\title{
Development of a New Binary Solvent System Using Ionic Liquids as Additives to Improve Rotenone Extraction Yield from Malaysia Derris sp.
}

\author{
Zetty Shafiqa Othman, ${ }^{1}$ Nur Hasyareeda Hassan, ${ }^{1}$ \\ Muhammad Rahimi Yusop, ${ }^{1,2}$ and Saiful Irwan Zubairi ${ }^{1,2}$ \\ ${ }^{1}$ School of Chemical Sciences and Food Technology, Faculty of Science and Technology, \\ Universiti Kebangsaan Malaysia (UKM), 43600 Bangi, Selangor, Malaysia \\ ${ }^{2}$ Advanced Medical and Dental Institute (AMDI), Universiti Sains Malaysia (USM), Bertam, \\ 13200 Kepala Batas, Pulau Pinang, Malaysia
}

Correspondence should be addressed to Saiful Irwan Zubairi; saiful-z@ukm.edu.my

Received 24 September 2014; Accepted 11 January 2015

Academic Editor: Kaustubha Mohanty

Copyright ( 2015 Zetty Shafiqa Othman et al. This is an open access article distributed under the Creative Commons Attribution License, which permits unrestricted use, distribution, and reproduction in any medium, provided the original work is properly cited.

\begin{abstract}
Rotenone is one of the prominent insecticidal isoflavonoid compounds which can be isolated from the extract of Derris sp. plant. Despite being an effective compound in exterminating pests in a minute concentration, procuring a significant amount of rotenone in the extracts for commercialized biopesticides purposes is a challenge to be attained. Therefore, the objective of this study was to determine the best ionic liquid (IL) which gives the highest yield of rotenone. The normal soaking extraction (NSE) method was carried out for $24 \mathrm{hrs}$ using five different types of binary solvent systems comprising a combination of acetone and five respective ionic liquids (ILs) of (1) [BMIM] Cl; (2) [BMIM] OAc; (3) [BMIM] NTf2; (4) [BMIM] OTf; and (5) [BMPy] Cl. Next, the yield of rotenone, $\%(\mathrm{w} / \mathrm{w})$, and its concentration $(\mathrm{mg} / \mathrm{mL})$ in dried roots were quantitatively determined by means of RP-HPLC and TLC. The results showed that a binary solvent system of [BMIM] OTf + acetone was the best solvent system combination as compared to other solvent systems $(P<0.05)$. It contributed to the highest rotenone content of $2.69 \pm 0.21 \%(\mathrm{w} / \mathrm{w})(4.04 \pm 0.34 \mathrm{mg} / \mathrm{mL})$ at $14 \mathrm{hrs}$ of exhaustive extraction time. In conclusion, a combination of the ILs with a selective organic solvent has been proven to increase a significant amount of bioactive constituents in the phytochemical extraction process.
\end{abstract}

\section{Introduction}

Rotenone is one of naturally isolating pesticides extracted mainly from the roots of Derris sp. in Asia, Lonchocarpus in South America, and several other legumes in the tropics region [1]. Derris elliptica is widely available as a local plant and it contains approximately $4 \%$ to $5 \%(\mathrm{w} / \mathrm{w})$ of rotenone in dried roots [2]. However, the rotenone content of Derris elliptica roots $(1.2 \%(\mathrm{w} / \mathrm{w}))$ collected from the state of Johor, Malaysia, has been found to be too low as compared to the one gathered from the other tropical countries (e.g., Amazonian Brazil and South Africa) [3]. Even though rotenone (Figure 1(a)) and other toxic constituents existing in the rotenoids resin exhibited a strong "knock-off" effect on the aquatic life and certain insects pest $[4,5]$, the problems are still present in getting a large amount of rotenone for the commercialized purposes. For that reason, additional study on the extraction process is needed to increase the content up to the profitable level of developing variety of biopesticides products.

In botanical research area, the extraction process is considered as a backbone of a research loop prior to bioactive constituent's purification and characterization [6]. It involves several important processing parameters (independent variables) which influences the efficacy of the extracts (e.g., toxicity). In fact, a conventional extraction process uses a variety of organic solvents which include from a highly polar to nonpolar solvents. However, organic solvents are mostly volatile, toxic, and flammable leading to several human risk, safety issues, and environmental problems [7]. Taking all 


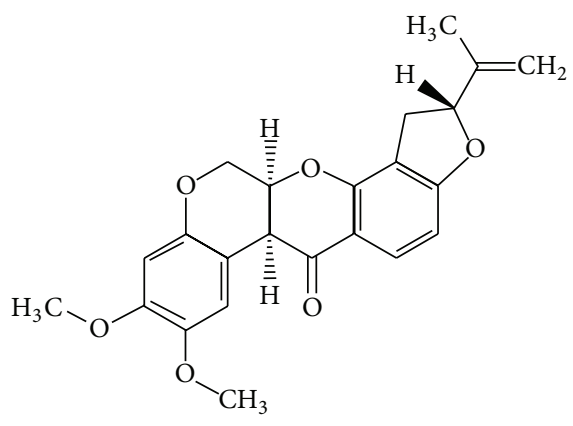

(a)

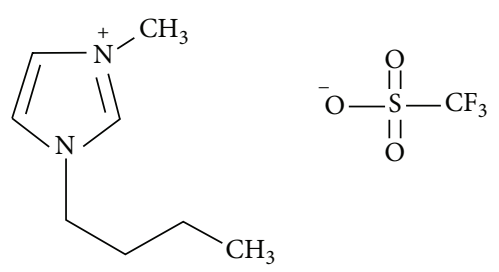

(b)

FIGURE 1: Molecular structure of (a) rotenone and (b) one of the selected ionic liquids: 1-butyl-3-methylimidazolium trifluoromethanesulfonate, [BMIM] OTf.

these into consideration, there have been several studies on the exploration of ionic liquids (ILs) compatibility as green solvents for phytochemicals extraction. Ionic liquids (ILs) are an organic salt in the liquid state under ambient temperature comprised of normally charge-stabilized organic cation paired with either organic or inorganic anions. It displays a wide range of unique properties such as high thermal stability, nonflammability, insignificant vapor pressure, and low chemical reactivity. In addition, ILs have fine tunable density, viscosity, polarity, and miscibility with other common solvents [8]. Commonly studied cations of ILs include 1-alkyl-3-methylimidazolium $\left([\mathrm{Cnmim}]^{+}\right)$, $\mathrm{N}$-alkylpyridinium $\left([\mathrm{Cnpy}]^{+}\right)$, tetraalkylphosphonium, and tetraalkylammonium. These cations can be paired with either organic or inorganic anions such as halide, acetate, hexafluorophosphate, tetrafluoroborate, trifluoromethanesulfonate, nitrate, or others. Innumerable molecular combinations are theoretically possible $\left(>10^{18}\right)$ [9] with various combinations of cations and anions affording distinct physiochemical properties such as melting point, polarity, viscosity, stability, salvation properties, and phase behavior. For that reason, the usage of ILs to aid the phytochemicals extraction process is considered as a great choice of increasing a significant amount of bioactive constituents. Besides, ILs can be used as green solvents to reduce the usage of toxic and volatile organic solvents, thus reducing environmental pollution [10]. One of the prominent ILs used in this study is shown in Figure 1(b).

However, ILs have relatively high polarity due to their charged and asymmetric structures [11], by which they do not have good affinity with weak-polar compounds and cause reduction in the distribution of weak-polar compounds in ILs phase. Although longer alkyl chain of ILs has lower polarity, its viscosity is large in accordance with the strong electrostatic and hydrogen bonding interaction between ions $[12,13]$. This drawback has impaired the mixing and transfer properties of extraction process by influencing the dissolution of compounds in ILs. In exchange, mixture of ILs and polar molecular solvents as extraction medium could be an effective approach to solve its flaws. Besides creating wide adjusted range of solvent polarity, hydrophobicity, hydrogen-bond acidity, and basicity $[14,15]$, the addition of miscible molecular solvents as cosolvent helps to break the microscopic hydrogen-bond network and the aggregation of ILs, which significantly reduce the viscosity and improve the mixing and transfer process in its mediated extraction $[16,17]$.

Rotenone extract has a great potential to be used as insecticidal products. However, the rotenone content is still low as compared to the commercial grade rotenoids resin [18]. Therefore, the objective of this research was to study the effects of five different ionic liquids on the yield of rotenone. The combination of ionic liquids with organic solvent as a binary solvent system would perhaps be one of the potential novel approaches to increase the yield of bioactive constituents of any botanical plants.

\section{Experimental Section}

2.1. Sample Collection and Preparation. Derris elliptica roots were first collected from Ladang 2, Faculty of Agriculture, Universiti Putra Malaysia (UPM), Malaysia. The collected roots (Figure 2(a)) were cleaned and cut into smaller parts prior to rapid drying. Cleaned parts of the roots were placed in the freezer to maintain its freshness and later dried using vacuum oven at the temperature of $28 \pm 2^{\circ} \mathrm{C}$ for $24 \mathrm{hrs}$. Once dried, the roots were ground into smaller particles size of approximately $0.86 \pm 0.20 \mathrm{~mm}$ (Figure 2(b)). The selected sieved ground samples were weighed prior to the normal soaking extraction process (NSE).

2.2. Preparation of Binary Solvent System. The binary solvent system comprises five selected colorless ILs which are listed as follows: (1) 1-butyl-3-methylimidazolium chloride, [BMIM] Cl; (2) 1-butyl-3-methylimidazolium acetate, [BMIM] OAc; (3) 1-butyl-3-methylimidazolium bis(trifluorosulfonyl)imide, [BMIM] $\mathrm{NTf}_{2}$; (4) 1-butyl-3-methylimidazolium trifluoromethanesulfonate, [BMIM] OTf; and (5) 1-butyl-1-methylpyrrolidinium chloride, [BMPy] $\mathrm{Cl}$. The binary solvent systems were prepared by adding $2 \mathrm{~mL}$ of respective ILs into a round bottom flask (with stopper) containing $18 \mathrm{~mL}$ of organic solvent (acetone) with a ratio of 1:9. To avoid any moisture absorption due to the hygroscopic properties of some ILs, the mixing process was carried out in the glove box. The mixtures were stirred by using magnetic stirrer for 5 to $6 \mathrm{hrs}$ to homogenize the combined solvents. The ratio of $1: 9$ was based on the exploratory experiment 


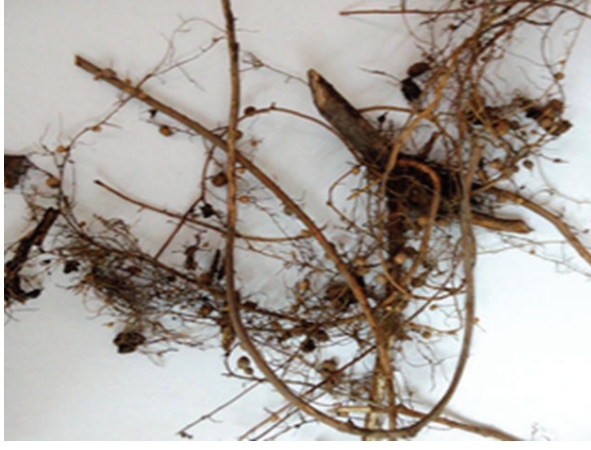

(a)

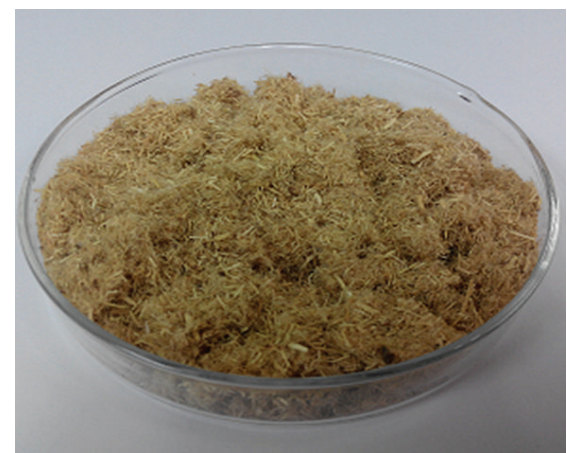

(b)

Figure 2: (a) Derris elliptica roots and (b) ground fine roots.

TAble 1: Processing parameters used in the rotenone extraction process.

\begin{tabular}{lc}
\hline \multicolumn{2}{c}{ Processing parameters } \\
\hline Solvent-to-solid ratio $(\mathrm{mg} / \mathrm{mL})$ & $10 \mathrm{~mL} / \mathrm{g}$ \\
Weight of raw materials $(\mathrm{g})$ & $0.50 \mathrm{~g}$ \\
Raw material particles size $(\mathrm{mm})^{*}$ & $0.86 \pm 0.20 \mathrm{~mm}$ \\
Temperature $\left({ }^{\circ} \mathrm{C}\right)^{*}$ & $28 \pm 2^{\circ} \mathrm{C}$ \\
ILs-to-acetone ratio & $1: 9$ \\
Optimum time of exhaustive extraction & $14 \mathrm{hrs}$ \\
\hline
\end{tabular}

${ }^{*}$ Error bars represent the standard deviation (SD) of triplicates $(n=3)$.

results (data not shown). The mixing of the ILs in acetone was considered homogeneous if no apparent residual appeared in the flask.

2.3. Normal Soaking Extraction. The extraction process was carried out in a room temperature at $28 \pm 2^{\circ} \mathrm{C}$ by using a combination of five different types of ILs and acetone with a mixing ratio of 1:9. The optimized parameters were utilized in accordance to Zubairi et al. [19] protocols as presented in Table 1 . The extraction process was carried out by soaking $0.50 \mathrm{~g}$ of dried roots in $10 \mathrm{~mL}$ of the respective solvent systems for $24 \mathrm{hrs}$ with solvent-to-solid ratio of $10 \mathrm{~mL} / \mathrm{g}(n=3)$. The liquid crude extract was collected twice at $14 \mathrm{hrs}$ and $24 \mathrm{hrs}$ prior to the reversed-phase high performance liquid chromatography (RP-HPLC) and thin layer chromatography (TLC) analysis.

2.4. Liquid Crude Extract Collection. The liquid crude extracts were collected at $14 \mathrm{hrs}$ and $24 \mathrm{hrs}$ and placed in the labeled vials. There were 18 samples in total of five different types of solvent used in three replicates $(n=3)$. Acetone was used as a control solvent. Next, the collected samples were placed in a freezer $\left(-18^{\circ} \mathrm{C}\right)$ to prevent any thermal degradation.

\subsection{Preparation of Fine Debris-Free Liquid Crude Extract.} The collected liquid crude extracts were diluted using analytical grade acetonitrile, Sigma-Aldrich, 95\% (v/v) with a dilution factor (DF) of 20 . Then, the extracts were filtered by using polytetrafluoroethylene (PTFE $-0.45 \mu \mathrm{m}$ pore size) vacuum filtration to remove any fine debris. A $2 \mathrm{~mL}$ vial was used to store the extracts prior to the qualitative and quantitative analysis.

2.6. Qualitative Analysis Using Thin Layer Chromatography. MERCK Silica gel 60 F254 TLC aluminium sheet was used as a stationary phase to observe the presence of rotenone in the liquid crude extract $(n=3)$. The migrations of rotenone markers were compared with rotenone standard, Dr. Ehrenstorfer $\mathrm{GmbH}, 93.80 \%$ (w/w). The markers and its migrated distance were visualized and determined, respectively, under UV light of $254 \mathrm{~nm}$ and $365 \mathrm{~nm}$ wavelengths. In the development chamber, chloroform and n-hexane were combined and utilized as a mobile phase system with a ratio of $70: 30$. The retardation factors $\left(R_{f}\right)$ of each extract were calculated by using

$$
\begin{aligned}
& \text { Retardation factor }\left(R_{f}\right) \\
& =\frac{\text { Migration distance of substance }}{\text { Migration distance of solvent front }} .
\end{aligned}
$$

2.7. Quantitative Analysis Using Reversed-Phase High Performance Liquid Chromatography. Approximately $21.80 \mathrm{mg}$ of rotenone standard of Dr. Ehrenstorfer GmbH, 93.80\% (w/w), was diluted with $50 \mathrm{~mL}$ of acetonitrile in a volumetric flask. The stock solution was filtered using Whatman filter paper number 2 with $8 \mu \mathrm{m}$ pore size. The quantitative analysis was carried out by using symmetry C18 $5 \mu \mathrm{L}$ column and waters with internal diameter of $4.6 \mathrm{~mm}$ and $150 \mathrm{~mm}$ length. The physical parameters involved in the RP-HPLC are as follows: (1) flow rate of $0.7 \mathrm{~mL} / \mathrm{min}$; (2) injection volume of $20 \mu \mathrm{L}$; (3) mobile phase of acetonitrile and deionized water with a ratio of $60: 40$; and (4) photodiode array detector (PDA) wavelength at $294 \mathrm{~nm}$.

\section{Results and Discussion}

3.1. Ionic Liquids Solubility (ILs) in Acetone. To enhance the rotenone extraction capacity, several ionic liquids were preliminarily selected. However, there was a drawback on its 


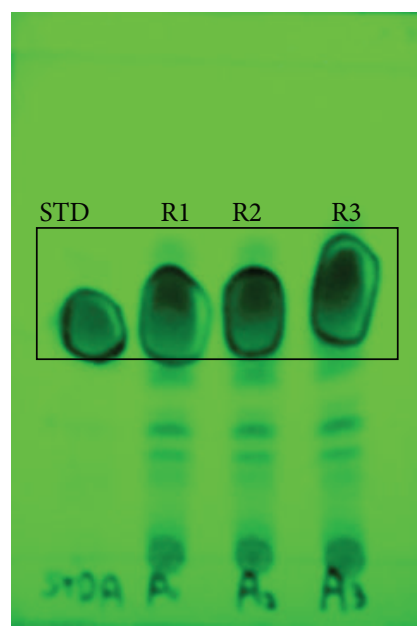

(a)

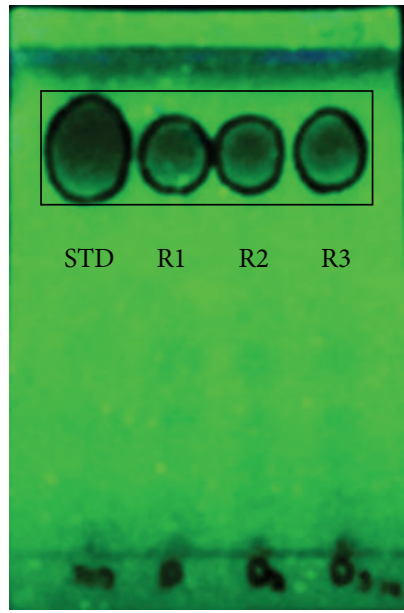

(d)

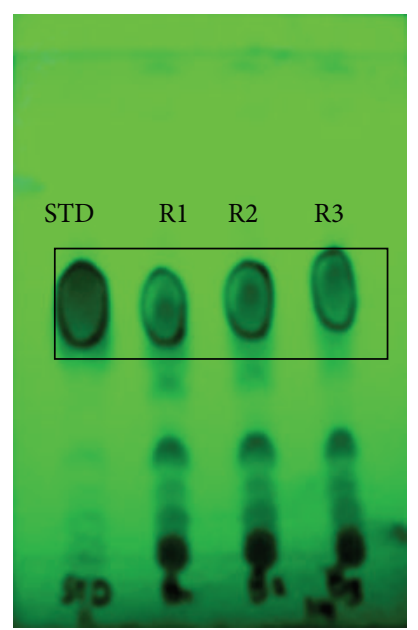

(b)

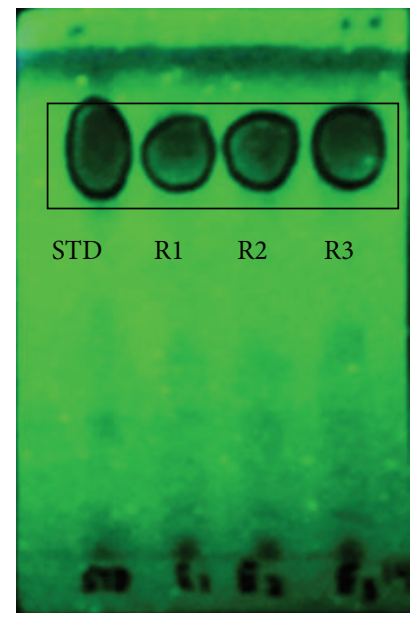

(e)

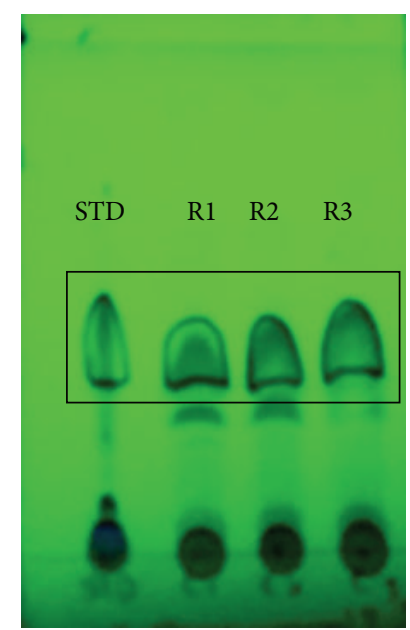

(c)

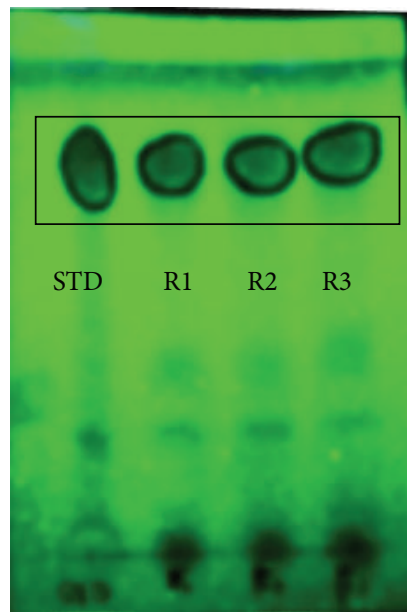

(f)

FIGURE 3: Visualization of rotenone migration markers of five different types of binary solvent systems used at 14 hrs of extraction. The markers were visualized on the alumina-based TLC plate under UV light of $254 \mathrm{~nm}$. (a) Acetone; (b) [BMIM] Cl; (c) [BMIM] Oac; (d) [BMIM] NTF2; (e) [BMIM] OTF; and (f) [BMP] Cl. The dark circled markers of STD and R (R1, R2, and R3) represent the rotenone standard and replication of each binary solvent systems used.

solubility with organic solvents. The solubility of the selected ILs in acetone was observed by a normal mixing with a ratio of $1: 9$. Meanwhile, an exploratory experiment has shown that the higher the amount of ILs used in the mixtures is, the higher the tendency of the ILs to produce undissolved solid residual is (data not shown). Out of five selected ILs, only 1-butyl-3-methylimidazolium acetate, [BMIM] OAc, 1-butyl3-methylimidazolium bis(trifluorosulfonyl)imide, [BMIM] $\mathrm{NTf}_{2}$, and 1-butyl-3-methylimidazolium trifluoromethanesulfonate, [BMIM] OTf, can be easily homogenized. However, 1-butyl-3-methylimidazolium chloride, [BMIM] $\mathrm{Cl}$, and 1butyl-1-methylpyrrolidinium chloride, [BMPy] $\mathrm{Cl}$, required a longer time to be homogenized (with the aid of heating at $80^{\circ} \mathrm{C}$ prior to mixing with acetone) which could possibly be due to their physical properties (solid form at ambient temperature) and a specific range of organic solvent polarity so that the solubility of the ILs in the organic solvent can be achieved. Theoretically, ionic liquids are miscible with an organic solvent of a medium to high dielectric constant $(\varepsilon)$ and become immiscible with a low dielectric constant $(\varepsilon)$ $[20,21]$. Thus, the solubility of all selected ILs was considered satisfactory as no apparent residual appeared in the flask due to the high dielectric constant of acetone that aids the solubility of both chemicals.

3.2. Qualitative Analysis on Rotenone Presence. The qualitative analysis of thin layer chromatography (TLC) was carried out to identify the existences of rotenone in the extracts of all binary solvent systems used. Figure 3 shows the images of rotenone markers visualized under UV light of $254 \mathrm{~nm}$. All samples exhibited a presence of rotenone markers in the extracts. Meanwhile, Table 2 shows the migration distance $(\mathrm{cm})$ and retardation factor $\left(R_{f}\right)$ of rotenone in a standard solution and liquid crude extracts. The results show that the rotenone $R_{f}$ value in a standard solution and all extracts 
TABLE 2: Qualitative analysis of rotenone via TLC on varies binary solvent systems.

\begin{tabular}{|c|c|c|c|c|}
\hline \multirow[t]{2}{*}{ Binary solvent system } & \multicolumn{2}{|c|}{$\begin{array}{l}\text { Rotenone migration } \\
\text { distance, Ds }(\mathrm{cm})^{\mathrm{b} *}\end{array}$} & \multicolumn{2}{|c|}{ Retardation factor $\left(R_{f}\right)^{*}$} \\
\hline & $14 \mathrm{hrs}$ & $24 \mathrm{hrs}$ & $14 \mathrm{hrs}$ & $24 \mathrm{hrs}$ \\
\hline Rotenone standard $\mathrm{a}^{\mathrm{a}}$ & $1.80 \pm 0.05$ & $2.60 \pm 0.03$ & $0.45 \pm 0.04$ & $0.65 \pm 0.03$ \\
\hline Control (acetone) & $2.03 \pm 0.06$ & $2.60 \pm 0.01$ & $0.51 \pm 0.02$ & $0.65 \pm 0.04$ \\
\hline [BMIM] $\mathrm{Cl}+$ acetone & $1.93 \pm 0.06$ & $2.20 \pm 0.02$ & $0.49 \pm 0.01$ & $0.55 \pm 0.01$ \\
\hline [BMIM] Oac + acetone & $1.53 \pm 0.06$ & $1.40 \pm 0.04$ & $0.39 \pm 0.01$ & $0.35 \pm 0.01$ \\
\hline [BMIM] NTF2 + acetone & $3.20 \pm 0.01$ & $2.17 \pm 0.06$ & $0.80 \pm 0.01$ & $0.54 \pm 0.01$ \\
\hline [BMIM] OTF + acetone & $3.20 \pm 0.08$ & $1.70 \pm 0.07$ & $0.80 \pm 0.08$ & $0.43 \pm 0.06$ \\
\hline [BMP] $\mathrm{Cl}+$ acetone & $3.10 \pm 0.00$ & $2.93 \pm 0.06$ & $0.78 \pm 0.00$ & $0.74 \pm 0.01$ \\
\hline
\end{tabular}

${ }^{a}$ Rotenone standard was prepared in acetone + ILs. ${ }^{b}$ Spotting volume (TLC spotting capillary tubes): $\sim 10 \mu \mathrm{L}$. Sample size: $\sim 25$ to $42 \mu \mathrm{g}$ rotenone/spot. ${ }^{*}$ Error bars represent the standard deviation (SD) of triplicates $(n=3)$.

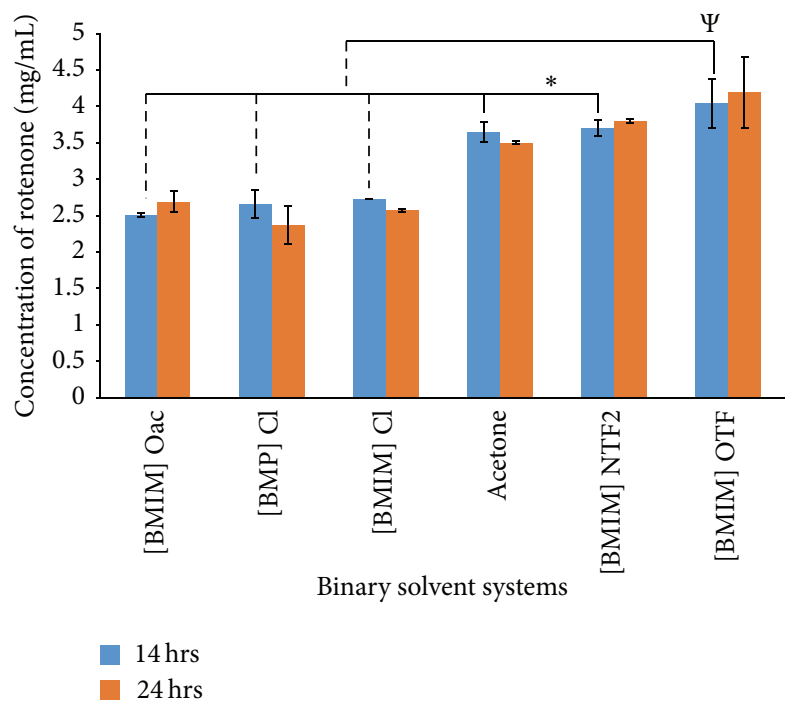

FIGURE 4: Concentration of rotenone $(\mathrm{mg} / \mathrm{mL})$ with respect to five different types of binary solvent systems. ${ }^{\Psi} P<0.05-[\mathrm{BMIMI}]$ OTF + acetone is the best binary solvent system to procure the highest rotenone concentration $(\mathrm{mg} / \mathrm{mL})$ at the optimum time of extraction process (14 hrs). ${ }^{*} P<0.05-[\mathrm{BMIMI}] \mathrm{NTF} 2+$ acetone and acetone extract produced high concentration as compared to the [BMIM] $\mathrm{Oac},[\mathrm{BMP}] \mathrm{Cl}$, and [BMIM] $\mathrm{Cl}$. Error bars represent the standard deviation (SD) of triplicates $(n=3)$.

(including control) were determined to be insignificantly different as compared to each other $(P>0.05)$. However, there were still a lot of impurities (unknown markers left behind rotenone) as presented in Figure 3. For that reason, a purification process of the liquid crude extracts via high vacuum pressure liquid chromatography (VLC) is highly recommended as to increase the accuracy of rotenone and its derivative compounds identification [22,23].

3.3. Quantitative Analysis on Rotenone Content. Figures 4 and 5 show the concentration $(\mathrm{mg} / \mathrm{mL})$ and yield of rotenone, $\%(\mathrm{w} / \mathrm{w})$, respectively, from five different types of binary solvent systems extracted at $14 \mathrm{hrs}$ and $24 \mathrm{hrs}$. The dependent

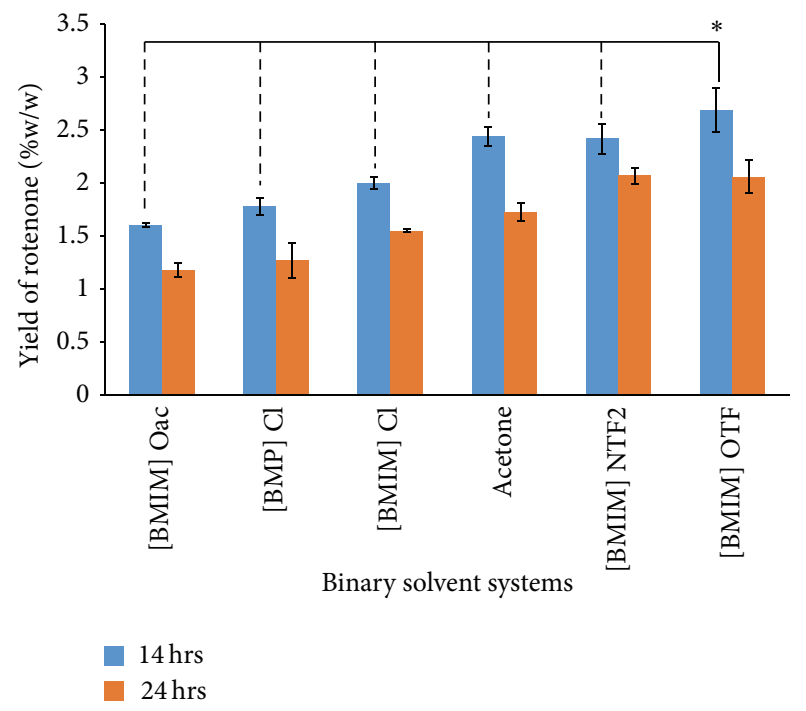

FIGURE 5: Yield of rotenone, $\%(\mathrm{w} / \mathrm{w})$ in dried roots with respect to five different types of binary solvent systems. ${ }^{*} P<0.05-[B M I M I]$ $\mathrm{OTF}+$ acetone is the best binary solvent system to procure the highest rotenone content in dried roots $(n=3)$ at the optimum time of extraction process (14 hrs). Error bars represent the standard deviation (SD) of triplicates $(n=3)$.

variables were calculated based on the external standard method of RP-HPLC. The retention time of rotenone in the standard solution and liquid crude extract was observed at 8.83 mins (Figure 6) and 8.82 mins (Figure 7), respectively. Overall, it was observed that the binary solvent system of 1-butyl-3-methylimidazolium trifluoromethanesulfonate, [BMIM] OTf, produced the highest rotenone concentration of $4.04 \pm 0.34 \mathrm{mg} / \mathrm{mL}$ and $4.19 \pm 0.48 \mathrm{mg} / \mathrm{mL}$ as compared to other ionic liquids and control solvent (acetone) $(P<0.05)$ at $14 \mathrm{hrs}$ and $24 \mathrm{hrs}$, respectively. Meanwhile, the highest yield of rotenone $(2.69 \pm 0.21 \%(\mathrm{w} / \mathrm{w})$ and $2.03 \pm 0.11 \%(\mathrm{w} / \mathrm{w})$ in dried roots) was determined in the 1-butyl-3-methylimidazolium trifluoromethanesulfonate, [BMIM] OTf, solvent system at $14 \mathrm{hrs}$ and $24 \mathrm{hrs}$, respectively. However, both of the reported previous studies of the optimized processing parameters and 


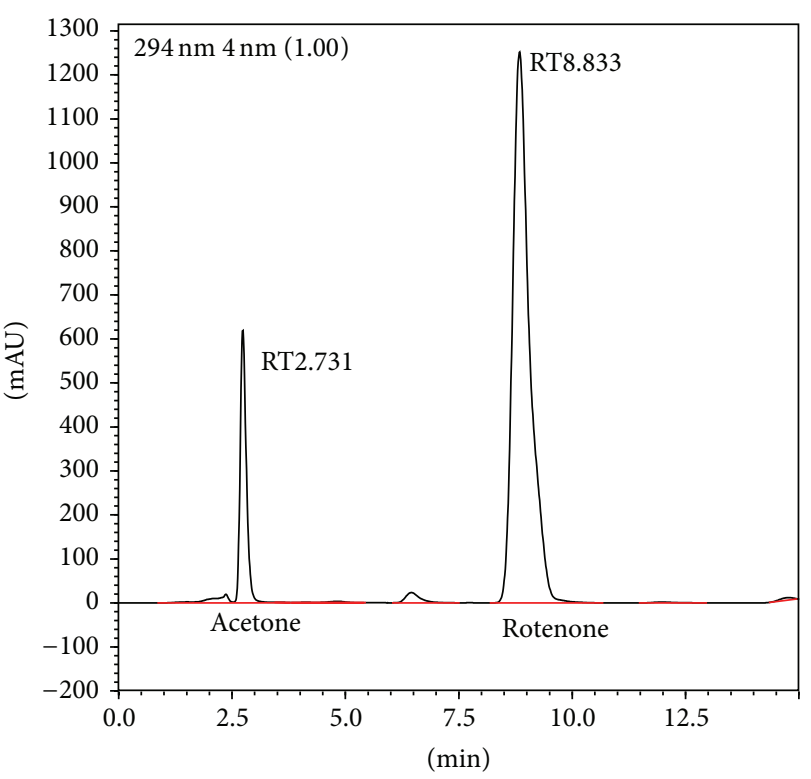

Figure 6: Chromatogram of rotenone standard.

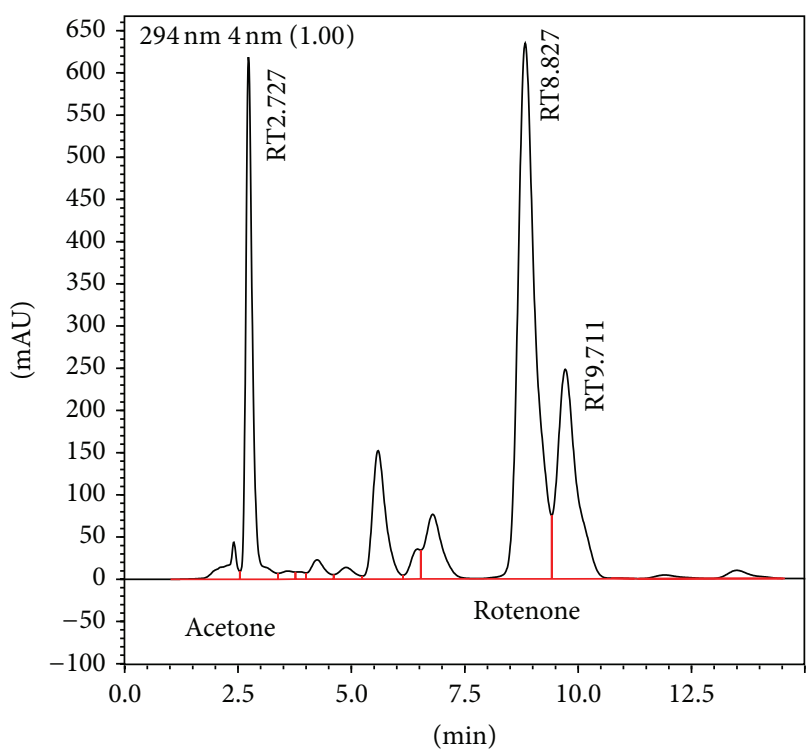

FIGURE 7: Chromatogram of [BMIM] OTF + acetone binary solvent system at $14 \mathrm{hrs}$.

the control extract (acetone) resulted in only $2.44 \pm 0.02 \%$ $(\mathrm{w} / \mathrm{w})$ [20] and $2.44 \pm 0.09 \%(\mathrm{w} / \mathrm{w})$ with a concentration of $3.65 \pm 0.13 \mathrm{mg} / \mathrm{mL}$, respectively. The results were approximately $24 \%$ lower than the yield of rotenone extracted using a combination of [BMIM] OTf + acetone $(P<0.05)$. This phenomenon appeared due to the large availability of the OTF anion (Figure 1(b)) which helps attracting more hydroxyl group of rotenone to form more hydrogen bonding [24, 25].

The significant increase of the rotenone content can be explained in several aspects which are as follows: (1) the susceptibility of rotenone functional groups against foreign charges and (2) the capacity of ILs in multiple charges environment. Rotenone compound as illustrated in Figure 1(a) is an acidic isoflavonoid compound that consists of ketonic chemical groups $(\mathrm{R}-\mathrm{C}(=\mathrm{O})-\mathrm{R})[26]$ which has the potential of interacting with intermediate polarity solvents. It can be easily dissolved in moderate polarity organic solvents (e.g., methanol, chloroform, and acetone) [27] and sparingly soluble in water [28]. For that reason, a combination of any ILs with moderate polarity of organic solvents would perhaps increase the chances of extracting high rotenone content. Previous study has shown that solubility of flavonoids and its derivatives can be increased by using ILs as the components are greatly an anion-dependent variable [24]. The anionic potency of both organic solvent and ILs in extracting large amount of bioactive compounds (e.g., rotenone) into the solvent systems is significantly undeniable as both chemicals facilitated the extraction process via salvation power and multiple interactions (e.g., hydrogen bonding, polarity, ionic/charge-to-charge, and $\pi-\pi, \pi-n)$ with the analytes [25, 29].

\section{Conclusions}

In conclusion, the best ionic liquid to aid the organic solvent (acetone) extraction system was 1-butyl-3-methylimidazolium trifluoromethanesulfonate, [BMIM] OTf. The selected binary solvent system has contributed to the highest rotenone content of $2.69 \pm 0.21 \%$ (w/w) with a concentration of $4.04 \pm 0.34 \mathrm{mg} / \mathrm{mL}$ at $14 \mathrm{hrs}$ (time of exhaustive extraction as reported in the previous study). The rotenone content was $24 \%$ higher than the optimized parameter of the acetone extract (control) $(P<0.05)$. Therefore, the addition of certain ionic liquids to the organic solvent will potentially give a significant increase to the amount of bioactive constituent in the phytochemical extraction process. Further study is required to optimize several processing parameters especially on the mixing ratio between the ILs and organic solvent in order to verify the increase of rotenone content as the solubility problem between both chemicals is relatively prominent.

\section{Conflict of Interests}

The authors declare that there is no conflict of interests regarding the publication of this paper.

\section{Acknowledgment}

The authors would like to thank the Ministry of Higher Education (MOHE), Malaysia, for providing financial support to this project (FRGS/2/2013/TK04/UKM/03/1 and GGPM2013-078).

\section{References}

[1] P. P. Hien, H. Gortnizka, and R. Kraemer, "Rotenone-potential and prospect for sustainable agriculture," Omonrice, vol. 11, pp. 83-92, 2003. 
[2] S. Gaby, Natural Crop Protection in the Tropics-DERRIS: Derris elliptica, D. malaccensis, D. uliginosa, Margraf Publishers GmbH Scientific books, Weikersheim, Germany, 1986.

[3] S. I. Zubairi, M. R. Sarmidi, and R. A. Aziz, "A preliminary study of rotenone exhaustive extraction kinetic from Derris elliptica dried roots using normal soaking extraction (NSE) method," Advances in Environmental Biology, vol. 8, no. 4, pp. 910-915, 2014.

[4] S. I. Zubairi, M. R. Sarmidi, and R. A. Aziz, "Biological activity on the extract of derris elliptica: an optimization approach to investigate the effect of processing parameters on mortality of artemia salina," Advances in Environmental Biology, vol. 8, no. 10, pp. 918-924, 2014.

[5] K. R. Ott, A Brief Review of its Chemistry, Environmental Fate, and the Toxicity of Rotenone Formulations, New Mexico Council of Trout Unlimited, 2008.

[6] S. Sasidharan, Y. Chen, D. Saravanan, K. M. Sundram, and L. Yoga Latha, "Extraction, isolation and characterization of bioactive compounds from plants' extracts," African Journal of Traditional, Complementary and Alternative Medicines, vol. 8, no. 1, pp. 1-10, 2011.

[7] P. A. Carson, Hazardous Chemicals Handbook, ButterworthHeinemann, Boston, Mass, USA, 2nd edition, 2002.

[8] M. G. Bogdanov, D. Petkova, S. Hristeva, I. Svinyarov, and W. Kantlehner, "New guanidinium-based room temperature ILS substituent and anion effect on density and solubility in water," Zeitschrift für Naturforschung B, vol. 65, no. 1, pp. 37-48, 2010.

[9] J. D. Holbrey and K. R. Seddon, "Ionic liquids," Clean Products and Processes, vol. 1, no. 4, pp. 223-236, 1999.

[10] X. Fu, S. Dai, and Y. Zhang, "Comparison of extraction capacities between ionic liquids and dichloromethane," Chinese Journal of Analytical Chemistry, vol. 34, no. 5, pp. 598-602, 2006.

[11] C. Reichardt, "Polarity of ionic liquids determined empirically by means of solvatochromic pyridinium $\mathrm{N}$-phenolate betaine dyes," Green Chemistry, vol. 7, no. 5, pp. 339-351, 2005.

[12] L.-L. Xie, A. Favre-Reguillon, X.-X. Wang et al., "Selective extraction of neutral nitrogen compounds found in diesel feed by 1-butyl-3-methyl-imidazolium chloride," Green Chemistry, vol. 10, no. 5, pp. 524-531, 2008.

[13] J. G. Huddleston, A. E. Visser, W. M. Reichert, H. D. Willauer, G. A. Broker, and R. D. Rogers, "Characterization and comparison of hydrophilic and hydrophobic room temperature ionic liquids incorporating the imidazolium cation," Green Chemistry, vol. 3, no. 4, pp. 156-164, 2001.

[14] B. R. Mellein, S. N. V. K. Aki, R. L. Ladewski, and J. F. Brennecke, "Solvatochromic studies of ionic liquid/organic mixtures," Journal of Physical Chemistry B, vol. 111, no. 1, pp. 131138, 2007.

[15] P. M. Mancini, G. G. Fortunato, and L. R. Vottero, "Molecular solvent/ionic liquid binary mixtures: designing solvents based on the determination of their microscopic properties," Physics and Chemistry of Liquids, vol. 42, no. 6, pp. 625-632, 2004.

[16] E. Gómez, B. González, Á. Domínguez, E. Tojo, and J. Tojo, "Dynamic viscosities of a series of 1-alkyl-3-methylimidazolium chloride ionic liquids and their binary mixtures with water at several temperatures," Journal of Chemical and Engineering Data, vol. 51, no. 2, pp. 696-701, 2006.

[17] A. Heintz, "Recent developments in thermodynamics and thermophysics of non-aqueous mixtures containing ionic liquids. A review," The Journal of Chemical Thermodynamics, vol. 37, no. 6 , pp. 525-535, 2005.
[18] S. I. Zubairi, M. R. Sarmidi, and R. A. Aziz, "Bio-active constituents of rotenoids resin extracted from Derris elliptica roots: comparison between local plant extract and SAPHYR (France) cube resin," Advances in Environmental Biology, vol. 8, no. 4, pp. 904-909, 2014.

[19] S. I. Zubairi, M. R. Sarmidi, and R. A. Aziz, "The effects of raw material particles size, types of solvents and solvent-To-Solid ratio on the yield of rotenone extracted from derris elliptica roots," Sains Malaysiana, vol. 43, no. 5, pp. 707-713, 2014.

[20] P. Bonhôte, A.-P. Dias, N. Papageorgiou, K. Kalyanasundaram, and M. Grätzel, "Hydrophobic, highly conductive ambienttemperature molten salts," Inorganic Chemistry, vol. 35, no. 5, pp. 1168-1178, 1996.

[21] C. F. Poole, B. R. Kersten, S. S. J. Ho, M. E. Coddens, and K. G. Furton, "Organic salts, liquid at room temperature, as mobile phases in liquid chromatography," Journal of Chromatography A, vol. 352, no. C, pp. 407-425, 1986.

[22] J. C. Touchstone, Practice of Thin Layer Chromatography, John Wiley \& Sons, New York, NY, USA, 1992.

[23] S. I. Zubairi, M. R. Sarmidi, and R. A. Aziz, "Identification of bio-active constituents from Derris elliptica liquid crude extract using liquid chromatographic method coupled with high vacuum pressure," Advances in Environmental Biology, vol. 8, no. 2, pp. 437-440, 2014.

[24] Z. Guo, B.-M. Lue, K. Thomasen, A. S. Meyer, and X. Xu, "Predictions of flavonoid solubility in ionic liquids by COSMO-RS: experimental verification, structural elucidation, and solvation characterization," Green Chemistry, vol. 9, no. 12, pp. 1362-1373, 2007.

[25] J. L. Anderson, J. Ding, T. Welton, and D. W. Armstrong, "Characterizing ionic liquids on the basis of multiple solvation interactions," Journal of the American Chemical Society, vol. 124, no. 47, pp. 14247-14254, 2002.

[26] F. Ahmad and H. Raji, Kimia hasilan semula jadi dan tumbuhan ubatan, Dewan Bahasa dan Pustaka, 1993.

[27] M. Cabizza, A. Angioni, M. Melis, M. Cabras, C. V. Tuberoso, and P. Cabras, "Rotenone and rotenoids in cubè resins, formulations, and residues on olives," Journal of Agricultural and Food Chemistry, vol. 52, no. 2, pp. 288-293, 2004.

[28] A. Dhaouadi, L. Monser, and N. Adhoum, "Removal of rotenone insecticide by adsorption onto chemically modified activated carbons," Journal of Hazardous Materials, vol. 181, no. 1-3, pp. 692-699, 2010.

[29] F.-Y. Du, X.-H. Xiao, and G.-K. Li, "Application of ionic liquids in the microwave-assisted extraction of trans-resveratrol from Rhizma Polygoni Cuspidati," Journal of Chromatography A, vol. 1140, no. 1-2, pp. 56-62, 2007. 

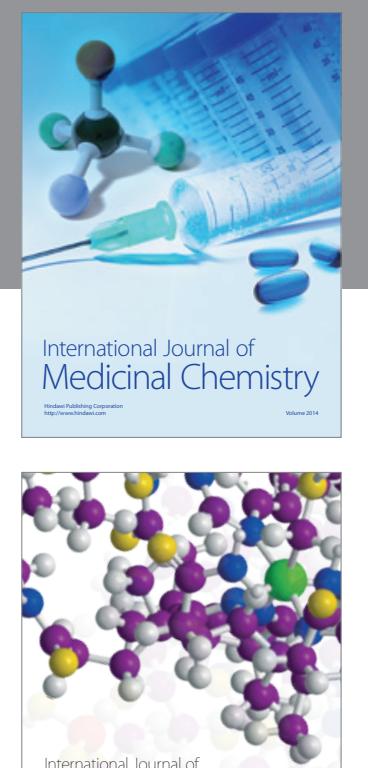

\section{Carbohydrate} Chemistry

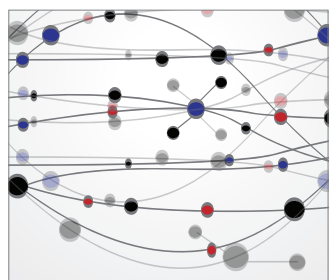

The Scientific World Journal
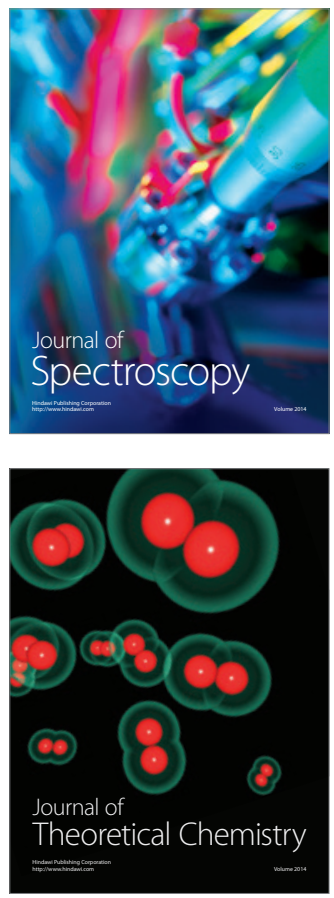
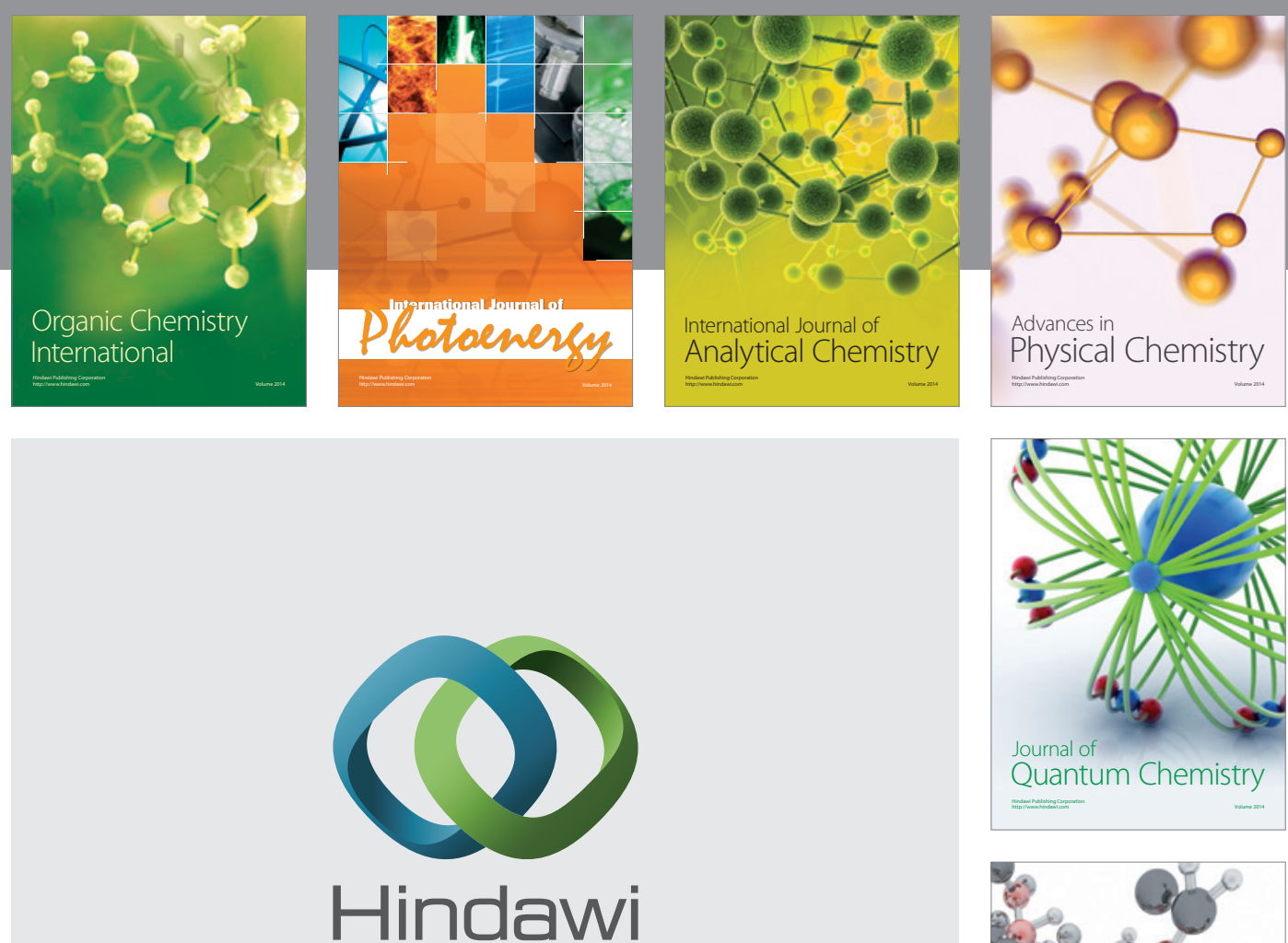

Submit your manuscripts at

http://www.hindawi.com

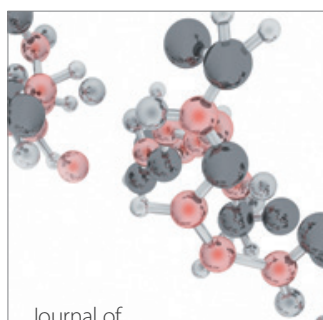

Analytical Methods

in Chemistry

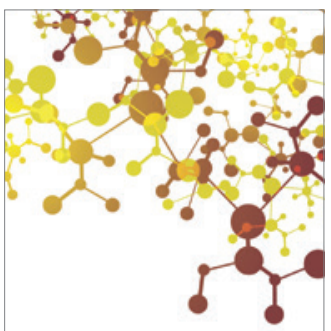

Journal of

Applied Chemistry

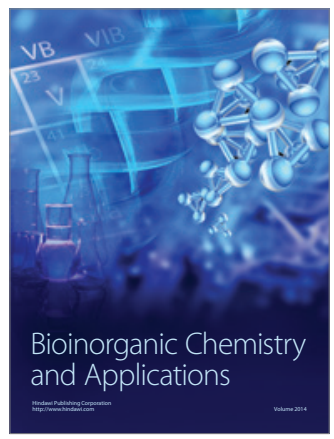

Inorganic Chemistry
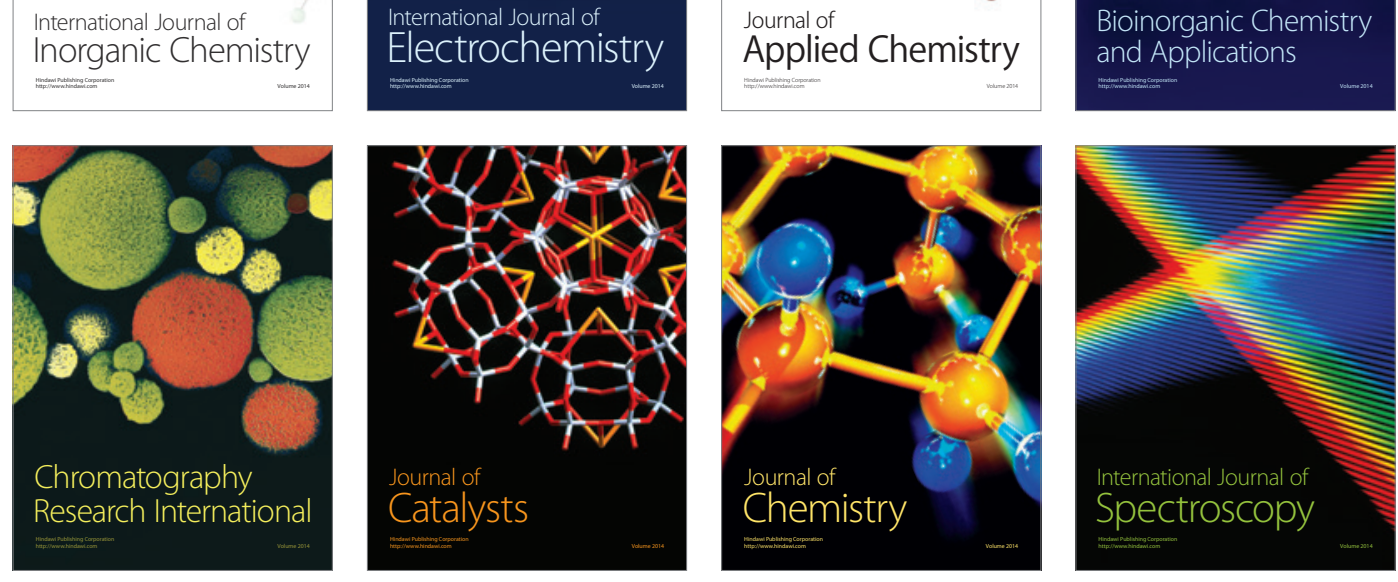